\title{
Convergence of Hermite-Fejér interpolation over roots of third-kind Chebyshev polynomials
}

\author{
Abedallah Rababah*
}

Department of mathematics and staticstic, Jordan University of Science and Technology, 22110 Irbid, Jordan

\section{A R T I C LE IN F O}

\section{Article history:}

Received 29 April 2016

Received in revised form

10 July 2016

Accepted 10 August 2016

\section{Keywords:}

Chebyshev polynomials

Hermite-Fejér interpolation

Functions of bounded variations

\section{Introduction}

For a given function, the Lagrange interpolation is, in many cases, not satisfactory. It is known that there exists a continuous function whose Lagrange interpolation diverges everywhere. So, there is a need to introduce additional conditions either related to the properties of the function or considering interpolating also derivatives-related data; this kind of interpolation is called the Hermite interpolation and will be considered in section 3 . If the function has, in particular, lack of derivative information, then we consider the derivatives equal to zeros; this kind of interpolation is called the Hermite-Fejér interpolation and will be considered in section 4. It is also known that when using the nodes of interpolation to be the roots of the orthogonal polynomials, then the convergence is faster and assured. The third-kind Chebyshev polynomials are introduced in section 2 . For more on these topics, see (Szegö, 1959). The rate of convergence of the Hermite-Fejér interpolation on the roots of the third-kind Chebyshev polynomials is given in section 5. Conclusions are given in section 6 .

Throughout this paper, we define, for $=$ $0,1, \ldots, 2 n-1$

$E_{r}(n, \theta)=\left\{k: \frac{r \pi}{2 n+1}<\left|\theta-\theta_{k}\right| \leq \frac{(r+1) \pi}{2 n+1}\right\}$

* Corresponding Author.

Email Address: rababah@just.edu.jo

https://doi.org/10.21833/ijaas.2016.12.010

2313-626X/C 2016 The Authors. Published by IASE

This is an open access article under the CC BY-NC-ND license

(http://creativecommons.org/licenses/by-nc-nd/4.0/)
Also, let $V_{f}[a, b]$ denote the total variation of the function $f(x)$ of bounded variation on the interval $[a, b]$.

\section{Third-kind Chebyshev polynomials}

The Chebyshev polynomials of the third kind, $V_{n}(x)$, are defined to be the orthogonal polynomials over the interval $[-1,1]$ with respect to the weight function

$w(x)=(1+x)^{1 / 2}(1-x)^{-1 / 2}$.

$V_{n}(x)$ is also defined by the trigonometric functions as follows:

$V_{n}(x)=\frac{\cos \left(n+\frac{1}{2}\right) \theta}{\cos \left(\frac{\theta}{2}\right)}, x=\cos \theta$

They satisfy the following orthogonality relations:

$$
\begin{gathered}
\int_{-1}^{1}(1+x)^{1 / 2}(1-x)^{-1 / 2} V_{n}(x) V_{m}(x) d x \\
=\left\{\begin{array}{cc}
\pi, & n=m \\
0, & n \neq m
\end{array}\right.
\end{gathered}
$$

They also satisfy the following recurrence relations:

$V_{n+1}(x)=2 x V_{n}(x)-V_{n-1}(x)$,

where

$V_{0}(x)=1, V_{1}(x)=2 x-1, n \geq 1$.

The first few terms can be calculated to be: 
$V_{2}(x)=4 x^{2}-2 x-1, \quad V_{3}(x)=8 x^{3}-4 x^{2}-4 x+1, \ldots$

The roots of $V_{n}(x)$ are given by:

$x_{k}=\cos \left(\frac{2 \mathrm{k}-1}{2 \mathrm{n}+1}\right) \pi, \quad k=1,2, \ldots, n$.

They are also a special case of the Jacobi polynomials and are related to them by the formula:

$$
\left(\begin{array}{c}
2 n \\
n
\end{array}\right) V_{n}(x)=2^{2 n} \quad P_{n}^{\left(-\frac{1}{2}, \frac{1}{2}\right)}(x)
$$

Where $P_{n}^{\left(-\frac{1}{2}, \frac{1}{2}\right)}(x)$ is the Jacobi polynomial of degree $n$.

They also satisfy the following Rodrigues' type formula:

$V_{n}(x)=\frac{(-2)^{n} n !}{(2 n) !}\left(\frac{1-x}{1+x}\right)^{\frac{1}{2}} \frac{d^{n}}{d x^{n}}\left\{\left(1-x^{2}\right)^{n}\left(\frac{1+x}{1-x}\right)^{\frac{1}{2}}\right\}$

They also satisfy the following second order differential equation:

$\left(1-x^{2}\right) y^{\prime \prime}-(2 x-1) y^{\prime}+n(n+1) y=0$.

The roots of $x_{k}, k=1,2, \ldots, n$ of $V_{n}(x)$ are all distinct real and lie in the interior of the interval. For more on the Chebyshev polynomials of the third kind, see (Doha et al., 2015, and Doha and AbdElhameed, 2014).

\section{Hermite interpolation}

Let $x_{k}, k=1,2, \ldots, n$ be nodes of interpolation from the interval $[-1,1]$, and let a function $f(x)$ be defined on $[-1,1]$. Let $y_{k}=f\left(x_{k}\right), k=1,2, \ldots, n$ and $y_{k}^{\prime}, k=1,2, \ldots, n$ be given numbers. Our aim is to construct a polynomial of degree $2 n-1$ that agrees with values of $f\left(x_{k}\right)$ and its derivatives agree with $y_{k}^{\prime}, k=1,2, \ldots, n$. It is known that the polynomial satisfying these conditions is the Hermite interpolation polynomial denoted by $H_{2 n-1}(x)$ and satisfies the following conditions:

$H_{2 n-1}\left(x_{k}\right)=f\left(x_{k}\right), H_{2 n-1}^{\prime}\left(x_{k}\right)=y_{k}^{\prime}, k=1,2, \ldots, n$.

It is the unique polynomial of degree $2 n-1$ that satisfies the conditions above. Suppose that there is another polynomial $P_{2 n-1}(x)$ of degree $2 n-1$ that satisfies the conditions above, and then the polynomial $D_{2 n-1}(x)=H_{2 n-1}(x)-P_{2 n-1}(x)$ is of degree $2 n-1$ at most that satisfies $D_{2 n-1}\left(x_{k}\right)=$ $0, D_{2 n-1}^{\prime}\left(x_{k}\right)=0, k=1,2, \ldots, n$. Thus $D_{2 n-1}(x)$ has roots of multiplicity 2 at each of the nodes $x_{k}$, $k=1,2, \ldots, n$. Since $D_{2 n-1}(x)$ is a polynomial of degree $2 n-1$ at most, thus, we must have $D_{2 n-1}(x)=0$. Therefore, $H_{2 n-1}(x)$ is unique.

The Hermite polynomial $H_{2 n-1}(x)$ is given explicitly by the formula:
$H_{2 n-1}(x)=\sum_{k=1}^{n} y_{k} h_{k}(x)+\sum_{k=1}^{n} y_{k}^{\prime} \hat{h}_{k}(x)$,

where

$h_{k}(x)=\left(1-\frac{w_{n}^{\prime \prime}\left(x_{k}\right)}{w_{n}{ }^{\prime}\left(x_{k}\right)}\left(x-x_{k}\right)\right) l_{k}^{2}(x), k=1,2, \ldots, n$,

$\hat{h}_{k}(x)=\left(x-x_{k}\right) l_{k}^{2}(x), k=1,2, \ldots, n$,

$w_{n}(x)=\left(x-x_{1}\right)\left(x-x_{2}\right) \ldots\left(x-x_{n}\right)$,

And $l_{k}(x), \quad k=1,2, \ldots, n$ are the Lagrange fundamental polynomials. The polynomials $h_{k}(x)$ and $\hat{h}_{k}(x)$, each of degree $2 n-1$, are called the fundamental polynomials of the first and second kind of the Hermite interpolation corresponding to the set of nodes $x_{k}, k=1,2, \ldots, n$.

If the function $f(x)$ is merely continuous on the interval $[-1,1]$, Fejér investigated the case of setting $H^{\prime}{ }_{2 n-1}\left(x_{k}\right)=y_{k}^{\prime}=0, k=1,2, \ldots, n$. In this case, the polynomial $H_{2 n-1}(x)$ is called the step polynomial or the Hermite-Fejér interpolating polynomial which behaves more regularly than the Lagrange interpolating polynomials as $n$ becomes large. In the next section this polynomial is considered when the nodes of interpolation are the roots of the third-kind Chebyshev polynomials.

\section{Hermite-Fejér interpolation}

These roots are used with the Hermite-Fejér interpolation as nodes of interpolation. We require the polynomial to take on given values at these roots and also to fix the values of its derivative at these roots. Given a function $f(x)$ on $[-1,1]$, we want to find a polynomial of lowest possible degree that satisfies the following properties:

$H_{2 n-1}\left(x_{k}\right)=f\left(x_{k}\right), H^{\prime}{ }_{2 n-1}\left(x_{k}\right)=0, k=1,2, \ldots, n$

The polynomial $H_{2 n-1}(x)$ is the Hermite-Fejér polynomial and is given by the following formula

$H_{2 n-1}(x)=\sum_{k=1}^{n} f\left(x_{k}\right) h_{k}(x)$

where

$h_{k}(x)=\left(1-\frac{V_{n}^{\prime \prime}\left(x_{k}\right)}{V_{n}{ }^{\prime}\left(x_{k}\right)}\left(x-x_{k}\right)\right) l_{k}^{2}(x)$,

$l_{k}(x), \quad k=1,2, \ldots, n$ are the Lagrange fundamental polynomials. Doing some simplifications, we get

$h_{k}(x)=\left(\left(1-x x_{k}\right)+\left(1-x_{k}\right)(x-\right.$

$\left.\left.x_{k}\right)\right)\left(\frac{V_{n}(x)}{\left(x-x_{k}\right)\left(n+\frac{1}{2}\right)}\right)^{2} c_{k}$,

where, see (Rababah, 2007),

$x_{k}=\cos \left(\frac{\cos ^{-1} x_{k}}{2}\right)$. 
When the function $f(x)$ is merely continuous on $[-1,1]$, and the nodes of interpolation $x_{k}, k=$ $1,2, \ldots, n$ are the roots of the Chebyshev polynomial of third kind, then the Hermite-Fejér interpolation polynomial $H_{2 n-1}(x)$ converges uniformly to $f(x)$ over every interval $[-1+\varepsilon, 1]$, see Bojanic and Cheng (1983), Al-Jarrah (1986), and Al-Jarrah and Rababah (1990).

\section{Rate of convergence}

In this section, we consider the Hermite-Fejér interpolation for functions of bounded variation on the roots of the Chebyshev polynomials of third kind $V_{n}(x)$. Let

$E_{n}(f, x)=H_{2 n-1}(x)-f(x)$.

We estimate the rate of convergence of $H_{2 n-1}(x)$ to $f(x)$ at points of continuity of $f(x)$. Let $x \in(-1,1)$, then we have

$$
\begin{aligned}
& \left|E_{n}(f, x)\right| \leq \sum_{k=1}^{n}\left|f\left(x_{k}\right)-f(x)\right| h_{k}(x) \\
& \leq \sum_{k=1}^{n} V_{f}\left[x-t_{k}, x+t_{k}\right] h_{k}(x)
\end{aligned}
$$

where,

$t_{k}=\left|x-x_{k}\right|$ and $x=\cos \theta, 0<\theta<\pi$

and

$x_{k}=\cos \theta_{k}, \theta_{k}=\left(\frac{2 \mathrm{k}-1}{2 \mathrm{n}+1}\right) \pi, k=1,2, \ldots, n$.

Thus, we have

$\left|E_{n}(f, x)\right| \leq \sum_{r=0}^{2 n-1} \sum_{k \in E_{r}(n, \theta)} V_{f}\left[x-t_{k}, x+t_{k}\right] \quad h_{k}(x)$

Since $\quad t_{k}=\left|x-x_{k}\right|=\left|\cos \theta-\cos \theta_{k}\right| \leq$ $\left|\theta-\theta_{k}\right| \leq \frac{\pi\left|V_{n}(x)\right|}{2 n+1}$ and $E_{r}(n, \theta)$ has at most two elements, we get

$$
\begin{aligned}
\sum_{k \in E_{0}(n, \theta)} V_{f}\left[x-t_{k}, x+t_{k}\right] & h_{k}(x) \\
& \leq 2 V_{f}\left[x-\frac{\pi\left|V_{n}(x)\right|}{2 n+1}, x+\frac{\pi\left|V_{n}(x)\right|}{2 n+1}\right]
\end{aligned}
$$

Since $t_{k} \leq \frac{\pi(r+1)}{2 n+1}$ and $h_{k}(x) \leq \frac{12 v_{n}^{2}(x)}{r^{2}}$, we have

$$
\begin{aligned}
\sum_{k \in E_{r}(n, \theta)} V_{f}\left[x-t_{k}, x+t_{k}\right] \quad h_{k}(x) \\
\leq \\
\leq \frac{24 V_{n}^{2}(x)}{r^{2}} V_{f}\left[x-\frac{\pi(r+1)}{2 n+1}, x\right. \\
\left.+\frac{\pi(r+1)}{2 n+1}\right] .
\end{aligned}
$$

Consequently,

$$
\begin{gathered}
\left|E_{n}(f, x)\right| \leq 2 \quad V_{f}\left[x-\frac{\pi\left|V_{n}(x)\right|}{2 n+1}, x+\frac{\pi\left|V_{n}(x)\right|}{2 n+1}\right]+ \\
24 V_{n}^{2}(x) \sum_{r=1}^{2 n-1} \frac{1}{r^{2}} V_{f}\left[x-\frac{\pi(r+1)}{2 n+1}, x+\frac{\pi(r+1)}{2 n+1}\right] . \\
\text { Let } p(t)=V_{f}[x-t, x+t], \text { then }
\end{gathered}
$$

$$
\begin{aligned}
& \sum_{r=1}^{2 n-1} \frac{1}{r^{2}} V_{f}\left[x-\frac{\pi(r+1)}{2 n+1}, x+\frac{\pi(r+1)}{2 n+1}\right]= \\
& \sum_{r=2}^{2 n} \frac{1}{(r-1)^{2}} p\left(\frac{\pi r}{2 n+1}\right) \leq 4 \sum_{r=2}^{2 n} \frac{1}{r^{2}} p\left(\frac{\pi r}{2 n+1}\right)
\end{aligned}
$$

$p(t)$ is a non-decreasing function and thus

$$
\int_{\frac{\pi r}{2 n+1}}^{\frac{\pi(r+1)}{2 n+1}} \frac{p(t)}{t^{2}} d t \geq p\left(\frac{\pi r}{2 n+1}\right) \int_{\frac{\pi r}{2 n+1}}^{\frac{\pi(r+1)}{2 n+1}} \frac{d t}{t^{2}} \geq p\left(\frac{\pi r}{2 n+1}\right) \frac{2 n+1}{\pi r(r+1)} .
$$

This can be rewritten as follows

$\frac{1}{r^{2}} p\left(\frac{\pi r}{2 n+1}\right) \leq \frac{\pi}{n} \int_{\frac{\pi r}{2 n+1}}^{\frac{\pi(r+1)}{2 n+1}} \frac{p(t)}{t^{2}} d t$.

Thus

$$
\sum_{r=2}^{2 n} \frac{1}{r^{2}} p\left(\frac{\pi r}{2 n+1}\right) \leq \frac{\pi}{n} \int_{\frac{2 \pi}{2 n+1}}^{\pi} \frac{p(t)}{t^{2}} d t \leq \frac{1}{n} \int_{1}^{n} p\left(\frac{\pi}{t}\right) d t
$$

The function $p\left(\frac{\pi}{t}\right)$ is non-increasing and thus we have

$$
\int_{1}^{n} p\left(\frac{\pi}{t}\right) d t \quad \leq \sum_{k=1}^{n} \quad p\left(\frac{\pi}{k}\right) .
$$

Thus

$$
\sum_{r=2}^{2 n} \frac{1}{r^{2}} p\left(\frac{\pi r}{2 n+1}\right) \leq \frac{1}{n} \sum_{k=1}^{n} p\left(\frac{\pi}{k}\right) .
$$

This discussion leads to the following formula for the estimate of the rate of convergence in the following theorem.

Theorem 1: Let $E_{n}(f, x)=H_{2 n-1}(x)-f(x), x \in$ $(-1,1)$, where $H_{2 n-1}(x)$ is the Hermite-Fejér interpolation to the function $f(x)$ of bounded variation on the roots of Chebyshev polynomials of third kind $V_{n}(x)$. At points of continuity $x \in$ $(-1,1)$, and for sufficiently large $n$, we have

$$
\begin{aligned}
& \left|E_{n}(f, x)\right| \leq \frac{96 V_{n}^{2}(x)}{\mathrm{n}} \sum_{k=1}^{n} V_{f}\left[x-\frac{\pi}{k}, x+\frac{\pi}{k}\right]+ \\
& 2 V_{f}\left[x-\frac{\pi\left|V_{n}(x)\right|}{2 n+1}, x+\frac{\pi\left|V_{n}(x)\right|}{2 n+1}\right],
\end{aligned}
$$

where $V_{f}[a, b] \quad$ is the total variation of $f(x)$ on $[a, b]$.

To check the precision of the estimate, we consider the Hermite-Fejér interpolation for even $n$ to the function $f(x)=x^{2}$ at $x=0$. 
We know that the Chebyshev polynomials of third kind $V_{n}(x)$ satisfy the following equalities at $x=0$ :

$V_{2 n}(0)=(-1)^{n}$, and $V_{2 n+1}(0)=(-1)^{n+1}$.

Thus

$E_{n}\left(x^{2}, 0\right)=\sum_{k=1}^{n} \frac{1-x_{k}+x_{k}^{2}}{x_{k}^{2}} \frac{V_{n}^{2}(0)}{\left(n+\frac{1}{2}\right)^{2}}$

Since $\frac{1-x_{k}+x_{k}^{2}}{x_{k}^{2}} \geq 1$, thus

$E_{n}\left(x^{2}, 0\right) \geq \sum_{k=1}^{n} \frac{1}{\left(n+\frac{1}{2}\right)^{2}} \geq \frac{1}{n}$.

Also, since the total variation $V_{f}[0, b]=b^{2}$ thus we get

$\left|E_{n}\left(x^{2}, 0\right)\right| \leq \frac{96}{\mathrm{n}} \quad \sum_{k=1}^{n} V_{f}\left[-\frac{\pi}{k}, \frac{\pi}{k}\right]$

$+2 V_{f}\left[-\frac{\pi}{2 n+1}, \frac{\pi}{2 n+1}\right]$

$\leq \frac{192}{\mathrm{n}} \quad \sum_{k=1}^{n} V_{f}\left[0, \frac{\pi}{k}\right]+4 \quad V_{f}\left[0, \frac{\pi}{2 n+1}\right]$

thus, we get

$\left|E_{n}\left(x^{2}, 0\right)\right| \leq \frac{192}{\mathrm{n}} \pi^{2} \quad \sum_{k=1}^{n} \frac{1}{k^{2}}+\frac{4 \pi^{2}}{(2 n+1)^{2}}$

We reach to the following theorem.

Theorem 2: $\operatorname{For} E_{n}(f, x)=H_{2 n-1}(x)-f(x), x \in$ $(-1,1)$, for $f(x)=x^{2}$, we have the following inequalities:

$\frac{1}{\mathrm{n}} \leq\left|E_{n}\left(x^{2}, 0\right)\right| \leq \frac{\mathrm{c}}{\mathrm{n}}$

for some positive constant c greater than 1 . This means that the precision of the estimate in Theorem 1 cannot be improved asymptotically.

\section{Conclusions}

In this paper, we have considered the HermiteFejér interpolation to functions of bounded variation on the roots of the third-kind Chebyshev polynomials as the nodes of interpolation. An estimate for the rate of convergence at the points of continuity for functions of bounded variations is given in Theorem 1. It is also shown that the rate of convergence cannot be improved asymptotically in Theorem 2 .

\section{Acknowledgement}

The author would like to thank the reviewers for invaluable comments.

\section{References}

Al-Jarrah R and Rababah A (1990). On the rate of convergence of Hermite-Fejér polynomials to functions of bounded variation on the zeros of certain Jacobi polynomials. Revista Colombiana De Matematicas, 24(1-2): 51-64.

Al-Jarrah R (1986). On the rate of convergence of Hermite-Fejér polynomials to functions of bounded variation on the Chebyshev nodes of the second kind. Dirasat Journal (Jordan), 13: 51-66.

Bojanic R and Cheng F (1983). Estimate of the rate of approximation of functions of bounded variation by Hermite-Fejér polynomials. In the $2^{\text {nd }}$ Edmonton Conference on Approximation Theory, CMS Conference Proceedings, 3: 5-17.

Doha E and Abd-Elhameed W (2014). Integrals of Chebyshev polynomials of third and fourth kinds: An application to solution of boundary value problems with polynomial coefficients. Journal of Contemporary Mathematical Analysis, 49(6): 296-308.

Doha E, Abd-Elhameed W, and Bassuony M (2015). On using third and fourth kinds Chebyshev operational matrices for solving Lane-Emden type equations. Romanian Journal of Physics, 60 (3-4): 281-292.

Rababah A (2007). Inequalities for the Jacobi Polynomials and their derivatives, International Journal of Pure and Applied Mathematics, 41(5): 697-701.

Szegö G (1959). Orthogonal polynomials. American Mathematical Society, New York, USA. 